\title{
Sorption/diffusion behaviour of anionic surfactants in polyacrylamide hydrogels: from experiment to modelling
}

\author{
A.J.M. Valente ${ }^{\text {a }}$, A.Ya. Polishchuk ${ }^{\text {b }, H . D . ~ B u r r o w s ~}{ }^{\mathrm{a}}$, \\ M.G. Miguel a, V.M.M. Lobo ${ }^{\text {a,* }}$ \\ a Department of Chemistry, University of Coimbra, 3004-535 Coimbra, Portugal \\ ${ }^{\mathrm{b}}$ Kompamid LLC, 18 Bazhova Street, Moscow 129128, Russia
}

Received 17 December 2002; received in revised form 17 December 2002; accepted 1 May 2003

\begin{abstract}
The sorption and diffusion processes of anionic surfactants with different chain length through polyacrylamide hydrogels with low swelling degree have been studied by electrical conductivity measurements. The multicomponent equilibrium equation has been used to model the sorption isotherms of different anionic surfactant in the hydrogels. Such isotherms show that initial rapid sorption of unimer surfactant into the membranes occurs, suggesting that nonfreezing water can be involved in these interactions. In aqueous solution, at concentrations near and above the critical micelle concentration an anti-co-operative region is found. The diffusion coefficients of the anionic surfactants inside the hydrogel matrix show that the mobility of diffusing surfactant entities is dependent on cross-linker concentration and chain length. The Cukier hydrodynamic model and the free volume theory as modified by Peppas and Reinhart were applied to explain the dependence of the diffusion coefficients of surfactant on surfactant concentration inside the hydrogel. The hydrodynamic model was applied with success to the more hydrophilic surfactant, sodium 1-octanesulfonate, showing that the diffusion coefficients, $D$, increase when the resistance to hydrodynamic medium decreases; when the surfactant chain length increases (sodium dodecyl sulfate and sodium 1-hexadecane sulphonate) the variation of $D$ with the free volume can only be understood considering the sieving effect produced by the surfactant inside gel.

(C) 2003 Elsevier Ltd. All rights reserved.
\end{abstract}

Keywords: Hydrogels; Polyacrylamide; Ionic surfactants; Diffusion coefficients

\section{Introduction}

The diffusion of solutes in polymer gels has been the subject of several studies, which have been reviewed in $[1,2]$. Much of the interest in this area has been driven by the dependence of many important industrial applications on diffusion, and on the possibilities of controlling this to obtain the desired flow characteristics. Surfactants are among the most important of such solutes for practical applications [3]. Though much work has been

\footnotetext{
* Corresponding author. Tel.: +351-239-828403/852080; fax: +351-239-827703.

E-mail address: vlobo@ci.uc.pt (V.M.M. Lobo).
}

done to characterise diffusion and permeation of surfactants in both neutral and ionic super-absorbent gels, as far as the authors know there is little work on the diffusion of ionic surfactants in hydrogels with very high polymer volume fractions. In this work we focus on surfactant diffusion in polymer gels. In general terms, the transport in polymeric matrices can be described in terms of chemical and/or frictional features. Effects of chemical interactions include the retardation of solute diffusion due to attractive forces. In some cases, chemical interactions dominate the diffusion process [4], whereas in other cases they are almost negligible. In contrast, solute diffusion will always be influenced by frictional effects, which include solvent effects, steric hindrance, hydrodynamic effects, etc. [5]. 


\begin{tabular}{|c|c|c|c|}
\hline \multicolumn{4}{|c|}{ Nomenclature } \\
\hline$A$ & area of the membrane & $F$ & integrated flux of surfactant; $F=J l$ \\
\hline$C$ & concentration of surfactant in polymer & $\varphi$ & polymer volume fraction \\
\hline$c$ & $\begin{array}{l}\text { concentration of surfactant in aqueous so- } \\
\text { lution }\end{array}$ & $\begin{array}{l}J \\
K\end{array}$ & $\begin{array}{l}\text { flux of surfactant through the membrane } \\
\text { partition coefficient }\end{array}$ \\
\hline$C^{\prime}$ & $\begin{array}{l}\text { concentration of solute adsorbed onto specific } \\
\text { sites of a polymer at saturation conditions }\end{array}$ & $\begin{array}{l}K^{\prime} \\
k, k_{1}, k_{\mathrm{c}},\end{array}$ & $\begin{array}{l}\text { equilibrium constant } \\
k_{2} \text { constants }\end{array}$ \\
\hline$c_{0}$ & $\begin{array}{l}\text { concentration of the surfactant in aqueous } \\
\text { solution prior to swelling experiments }\end{array}$ & $\begin{array}{l}Q \\
r_{\mathrm{s}}\end{array}$ & $\begin{array}{l}\text { polymer swelling degree } \\
\text { radius of the solute }\end{array}$ \\
\hline$c_{\infty}$ & $\begin{array}{l}\text { concentration of the surfactant in aqueous } \\
\text { solution swelling experiments }\end{array}$ & $\begin{array}{l}\rho \\
R^{2}\end{array}$ & $\begin{array}{l}\text { polymer density } \\
\text { correlation coefficient }\end{array}$ \\
\hline$\xi$ & mesh size of the hydrogel & $s$ & standard deviation \\
\hline$D$ & diffusion coefficient of surfactant in polymer & SE & standard error $(95 \%$ confidence interval) \\
\hline$D_{0}$ & $\begin{array}{l}\text { diffusion coefficient of surfactant in aqueous } \\
\text { solution }\end{array}$ & $\begin{array}{l}t \\
T\end{array}$ & $\begin{array}{l}\text { time } \\
\text { temperature }\end{array}$ \\
\hline$D_{\mathrm{i}}$ & integral diffusion coefficient & $V$ & volume of aqueous solution \\
\hline & density of surfactant in aqueous solution & $V_{P}$ & volume of membrane \\
\hline
\end{tabular}

In this paper we report the integral diffusion coefficients of anionic surfactants of different chain length in polyacrylamide membranes with polymer volume fractions that vary from 0.20 to 0.60 . In these systems, the frictional effects dominate and the chemical interactions, if they exist, are normally negligible. An approach will be made using theories for homogeneous hydrogels to discuss the experimental results on the basis of different features of the systems, such as mobility behaviour of unimers and aggregates (solute size), conformation of the polymer chain in the gel, steric hindrance, etc.

\section{Experimental}

\subsection{Preparation of the samples}

Sodium dodecyl sulfate (SDS), sodium octyl 1-sulfonate (SOS) and sodium 1-hexadecane sulfonate (SHS) were purchased from Sigma (Spain) and Lancaster (France). To check the effects of unimer and micelles on diffusion, the surfactant concentration range was chosen to contain the critical micelle concentration $(\mathrm{cmc})$ in the middle, and to span approximately two orders of mag- nitude. The aqueous solutions of these compounds were prepared using bi-distilled water of conductivity (1.2 \pm $0.4) \times 10^{-4} \Omega^{-1} \mathrm{~m}^{-1}$.

Acrylamide (AAm), $N, N^{\prime}$-methylene-bis-acrylamide (MBAAm) and sodium persulfate were purchased from Riedel-de-Haen. The polyacrylamide gels were prepared by free radical co-polymerisation of monomers in aqueous solutions. The amount of the cross-linker (MBAAm) and the initiator sodium persulfate (at a percentage $0.1 \%(\mathrm{wt} / \mathrm{v}))$ were added to acrylamide solution (5 M) and stirred until total homogenisation was observed; the pre-gel solution was placed inside two glass sheets, separated by a plastic rubber gasket; spring clips were used to hold the glass sheets together. The mould was placed in an oven at $50^{\circ} \mathrm{C}$ for $2 \mathrm{~h}$. After this, the gel membrane obtained was removed from the gasket and washed with a large excess of distilled water for two days. After that the membrane was placed between two plastic sheets, and stored inside a dessiccator at about $98 \%$ relative humidity. Table 1 shows the composition of the PAAm membranes.

The degree of swelling of the samples $\left(Q=w / w_{0}\right)$ was estimated from the weights of dry polyacrylamide (PAAm), $w_{0}$, and of swollen sample, $w$, and is equal to

Table 1

Parameters characterising composition and equilibrium water content in polyacrylamides at 25 and $50{ }^{\circ} \mathrm{C}$

\begin{tabular}{|c|c|c|c|c|c|}
\hline \multirow[t]{2}{*}{ Gel } & \multirow{2}{*}{$\begin{array}{l}\text { MBAAm/AAm } \\
(\mathrm{mol} \mathrm{ratio} / \%)\end{array}$} & \multicolumn{2}{|l|}{$T=25^{\circ} \mathrm{C}$} & \multicolumn{2}{|l|}{$T=50^{\circ} \mathrm{C}$} \\
\hline & & $Q(s)(\mathrm{w} / \mathrm{w})$ & $\rho(s)\left(\mathrm{g} \mathrm{cm}^{-3}\right)$ & $Q(s)(\mathrm{w} / \mathrm{w})$ & $\rho(s)\left(\mathrm{g} \mathrm{cm}^{-3}\right)$ \\
\hline PA-I & 0.003 & $12.8(0.1)$ & $0.36(0.03)$ & $12.33(0.01)$ & $1.04(0.09)$ \\
\hline PA-II & 0.007 & $9.34(0.08)$ & $0.24(0.01)$ & $9.74(0.04)$ & $1.06(0.04)$ \\
\hline PA-III & 0.07 & $4.02(0.07)$ & $0.20(0.01)$ & $4.07(0.01)$ & $1.10(0.01)$ \\
\hline
\end{tabular}

$Q=$ degree of swelling of the gels; $\rho=$ polymer density; $s=$ standard deviation of 24 independent measurements. 
the volume ratio of the samples in the different stages. The value of $w$ was measured for samples (approximately $1 \mathrm{~cm}^{2}$ ), after they had been immersed for at least 2 weeks in water or surfactant solution. The approach to equilibrium was controlled gravimetrically (ADA analytical balance, with a resolution of $0.1 \mathrm{mg}$ ). Surfactants have little effect on gel swelling degree [6]. Each experiment was repeated at least three times.

\subsection{Sorption and desorption experiments}

The concentration of surfactant sorbed by the membrane, $C$, was calculated by measuring the concentration of surfactant in the aqueous solution prior to $\left(c_{0}\right)$ and after $\left(c_{\infty}\right)$ the swelling experiments, using the expression

$C=\left(c_{\infty}-c_{0}\right) V / V_{P}$

where $V$ and $V_{P}$ are the volumes of the aqueous solution and membrane sample, respectively.

The membranes, which had previously been kept in equilibrium with water, were then immersed without stirring in the surfactant solution for 2 weeks until they attained equilibrium. The approach to equilibrium was monitored gravimetrically. Experiments were carried out in triplicate either at 25 or $50{ }^{\circ} \mathrm{C}$ (in the experiments with the SHS). The volume of samples was measured using a picometer with a resolution of $10 \mu \mathrm{m}$. Desorption experiments were carried out in a similar way: the polymeric membranes, after they had reached equilibrium in electrolyte solution, were immersed in a known volume of water for approximately 2 weeks. The amount of surfactant desorbed, as determined by conductivity (using a YSI 3200 conductivity instrument, with a cell constant of $0.1 \mathrm{~cm}^{-1}$ ), was then calculated.

\subsection{Permeability technique}

Permeability of surfactant in PAAm gels was measured using a cell similar to that previously reported [7]. This consists of two compartments filled with surfactant solution, compartment $\mathrm{A}$, and water, compartment $\mathrm{B}$, respectively. The hydrogel membrane (M), previously swollen in water up to equilibrium, was placed between the two compartments. Silicone was used to seal the membrane to ensure hermetic interfaces. The surfactant flux through the membrane was monitored by measuring the conductivity using a YSI 3200 apparatus. The conductivity system was calibrated after each experiment. Identical conditions were used for calibration and permeability experiments. During each experiment, the solutions in the compartments A and B were maintained at a constant temperature of 25 or $50{ }^{\circ} \mathrm{C}$ in a thermostatic bath (Velp Scientifica). The data were read at 2 min intervals over the time interval necessary to reach a surfactant concentration in compartment $B\left(c_{\mathrm{B}}\right)$ of ap- proximately two orders of magnitude lower than that in compartment $\mathrm{A}$ (i.e. $c_{\mathrm{A}} / c_{\mathrm{B}}>100$ ). For maximum precision, the experimental conductivity data used to calculate the integral diffusion coefficients were taken from at least 300 points under steady-state conditions. Both solutions were stirred magnetically at $220 \mathrm{rpm}$. The permeability cell has a working volume $V=200 \mathrm{~cm}^{3}$ and a working area of the membrane $A=1.5394 \mathrm{~cm}^{2}$.

The steady-state flux, $J$, was calculated from

$J=(V / A)(\mathrm{d} c / \mathrm{d} t)$

prior to calculation of diffusion coefficients of surfactants assuming Fickian diffusion and initial and boundary conditions

$C(0, t)=K c ; \quad C(l, t)=0, \quad C(x, 0)=0$

where $C$ and $c$ are the initial concentration of the electrolyte inside the membrane and in aqueous solution, respectively, $K$ is the partition coefficient, $l$ is the thickness of the membrane and $t$ is the time.

\section{Results and discussion}

\subsection{Sorption isotherms}

Figs. 1-3 show the sorption isotherms of surfactants with different carbon-chain length, SOS, SDS and SHS respectively, in water-swollen polyacrylamide membranes with different degrees of cross-linking. Note that all studies with SHS were done at $50{ }^{\circ} \mathrm{C}$ because of the higher Krafft point of this surfactant.

The concentration range used in the sorption experiments of the three different surfactants was chosen in the region of the $\mathrm{cmc}$ of each surfactant: $(0.1 \times \mathrm{cmc})<$ $c<(10 \times \mathrm{cmc})$. We may conclude, from Figs. $1-3$, that the sorption of surfactants in these gels depends on chain length as well as on the water content inside gels.

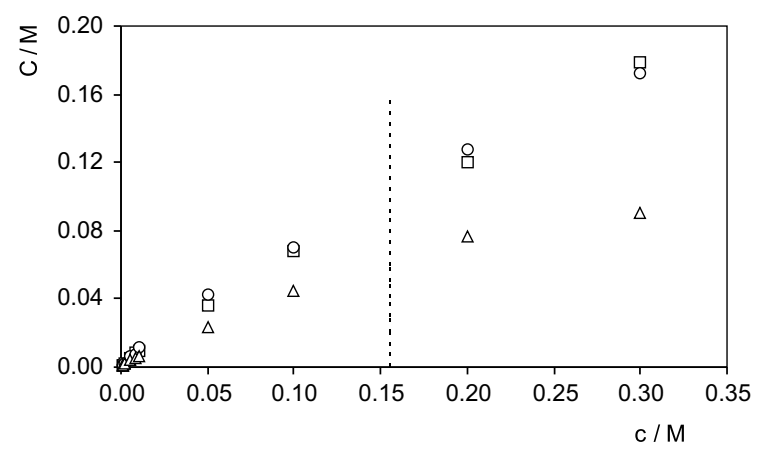

Fig. 1. Sorption isotherms of SOS at $25{ }^{\circ} \mathrm{C}$ in polyacrylamide membranes with different percentages of cross-linking: $(\square)$ $0.3 \%$; (O) $0.7 \%$; $\triangle$ ( $7.0 \%$. The dashed line shows the $\mathrm{cmc}$ in aqueous solution. 


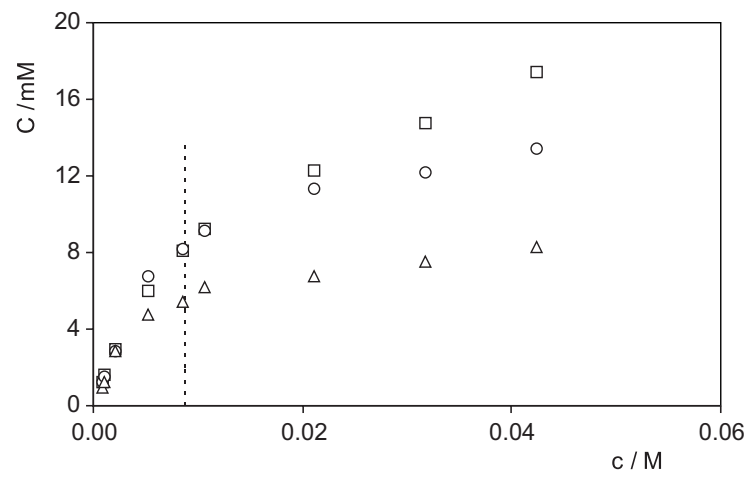

Fig. 2. Sorption isotherms of SDS at $25^{\circ} \mathrm{C}$ in polyacrylamide membranes with different percentages of cross-linking: $(\square)$ $0.3 \%$; (O) $0.7 \%$; $\triangle) 7.0 \%$. The dashed line shows the $\mathrm{cmc}$ in aqueous solution.

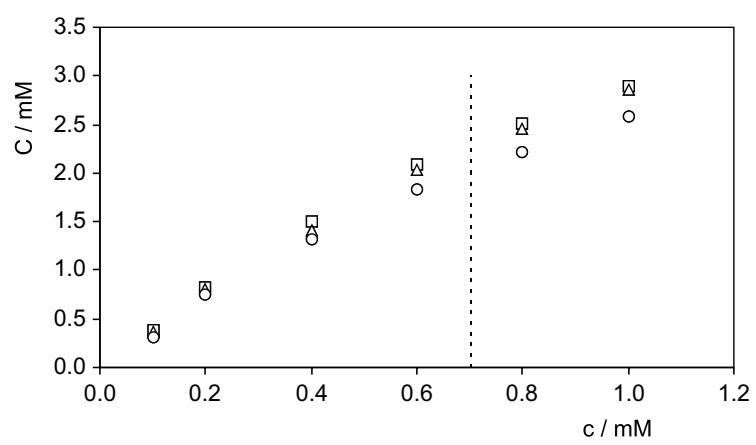

Fig. 3. Sorption isotherms $\left(50{ }^{\circ} \mathrm{C}\right)$ of SHS at $50{ }^{\circ} \mathrm{C}$ in polyacrylamide membranes with different percentages of crosslinking: $(\square) 0.3 \%$; (O) $0.7 \%$; $\triangle$ ) $7.0 \%$. The dashed line shows the $\mathrm{cmc}$ in aqueous solution.

Although the hydrophilic character of gel becomes less important for more hydrophobic surfactant systems, all isotherms suggest that the free volume of the polymeric matrix also plays an important role on the sorption mechanism since the distribution coefficient $(K=C / c)$ decreases with the decrease of percentage of water uptake.

For each surfactant system sorption is found to depend upon both the available water free volume and on micelle/unimer equilibrium at aqueous solution. This is supported by the fact that with all three surfactants, real effects of the cross-linker on the surfactant sorption are only observed at concentrations in the region of or above the critical micelle concentration of surfactants in aqueous solution ( $c=\mathrm{cmc}$, see dashed lines in Figs. 13 ). That is, at concentrations below cmc, the sorption of unimers by the gels is not cross-linker concentrationdependent. The analysis of Figs. 1-3 also shows that some competition between surfactant molecules (unimers) and hydrogel and/or surfactant/surfactant may occur. A possible approach is to treat competing equilibrium using a multiple equilibrium model [8]. This phenomenological approach can be mathematical expressed in the same way as that of the multicomponent isotherm; that is in a linear form

$\frac{c}{C}=\frac{c}{C^{\prime}}+\frac{1}{K^{\prime} C^{\prime}}$

where $C^{\prime}$ represents the concentration of solute adsorbed in specific sites of a polymer at saturation conditions, and $K^{\prime}$ is the corresponding equilibrium constant.

Table 2 shows the parameters $\left(C^{\prime}\right.$ and $\left.K^{\prime}\right)$ that best fit the experimental results of sorption isotherms. The fitting parameters were obtained using Origin 6.0 software taking a confidence interval of $95 \%$.

From the fitting parameters given in Table 2, we may conclude that with increasing surfactant carbon-chain length and decreasing water swelling there is a decrease of $C^{\prime}$, which can be due to inhibition by steric hindrance to possible further adsorption at specific sites, and an increase of $K^{\prime}$. In fact, $K^{\prime}$ values drastically increase on going from SOS to SHS, showing the highest value in the later systems, even though the experiments with SHS were carried out at higher temperature. Analysing the experimental results for each system surfactant/gels we also may observe that values of $K^{\prime}$ change with the gel properties. The analysis of sorption isotherms also suggests that initially there is a non-co-operative followed by an anti-co-operative region. This kind of interactions is common, as for example, in hydrophobically modified water-soluble polymers [3, p. 227-30]. In order to find an explanation we must bear in mind that the nature of the water within the hydrogel matrix is known to affect the

Table 2

Fitting parameters of the sorption isotherms of SOS, SDS and SHS in polyacrylamide gels with different degrees of crosslinking, according to Eq. (4)

\begin{tabular}{llll}
\hline \multirow{2}{*}{ Gel } & \multicolumn{2}{l}{ Fitting parameters } & \multirow{2}{*}{$R^{2}$} \\
\cline { 2 - 3 } & $C^{\prime}(\mathrm{SE})(\mathrm{M})$ & $K(\mathrm{SE})$ & \\
\hline SOS & & & 0.997 \\
I & $0.388(0.100)$ & $2.60(0.97)$ & 0.997 \\
II & $0.494(0.065)$ & $1.82(0.34)$ & 0.998 \\
III & $0.189(0.015)$ & $3.15(0.43)$ & \\
SDS & & & 0.995 \\
I & $0.0229(0.001)$ & $67(7)$ & 0.995 \\
II & $0.0156(0.0004)$ & $134(11)$ & 0.991 \\
III & $0.0087(0.0002)$ & $222(22)$ & 0.999 \\
SHS & & & 0.999 \\
I & $0.0078(0.0005)$ & $599(57)$ & 0.998 \\
II & $0.0084(0.0008)$ & $518(67)$ & $568(86)$ \\
III & $0.0071(0.0008)$ & 568 & \\
\hline
\end{tabular}

$\mathrm{SE}=$ standard error $\left(95 \%\right.$ confidence interval); $R^{2}=$ correlation coefficient. 
transport and sorption of salts through the membrane (e.g. $[9,10]$ ); within a hydrogel membrane, a continuum of states exists between "freezing" and "non-freezing" water [11], which have different solvent features. As a consequence the non-co-operative region does not result from interaction between the ionic surfactant and the polymer, but involves non-freezing water, which can have a structuring effect on the surfactant due to its highly non-polar carbon chain [12]. This means that the unimers, after being sorbed by the hydrogel, can themselves act as "main" sites of sorption, consequently forming aggregates.

\subsection{Permeation of surfactants through hydrogels}

Figs. 4-6 show the variation of the flux of surfactant, $F(=J l)$, through hydrogel as a function of the concentration, $c$. The flux of surfactant increases with surfactant concentration, for all the PAAm systems, showing that essentially all the surfactant is free to move inside gels. This shows that the equilibrium between unimers and/or

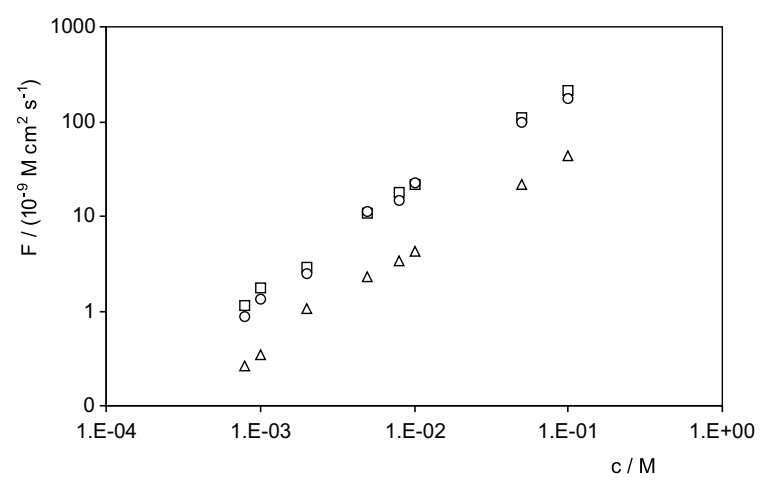

Fig. 4. Dependence of the SOS flux, $F$, on the concentration, at $25^{\circ} \mathrm{C}$, in hydrogels with different degrees of cross-linking: $(\square)$ $0.3 \%$; (O) $0.7 \%$; $\triangle$ ) $7.0 \%$.

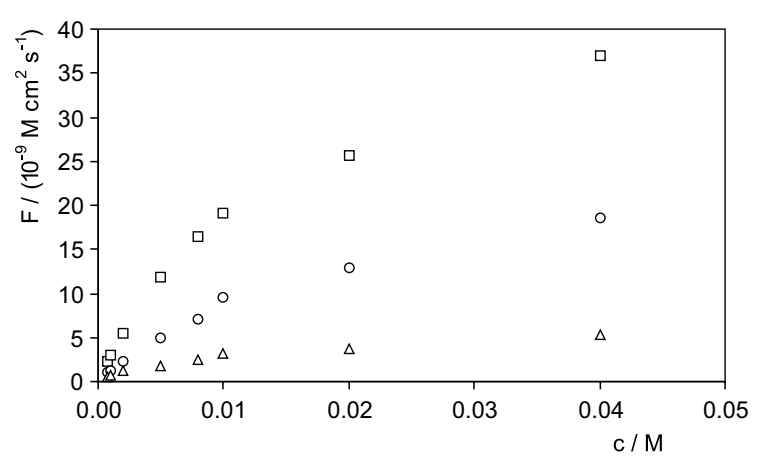

Fig. 5. Dependence of the SDS flux, $F$, on the concentration, at $25^{\circ} \mathrm{C}$, in PAAm with different degrees of cross-linking: $(\square)$ $0.3 \%$; (O) $0.7 \%$; $\triangle$ ) $7.0 \%$.

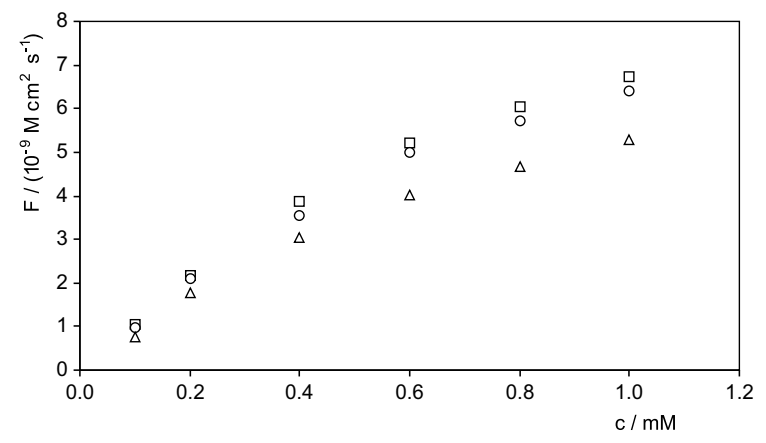

Fig. 6. Dependence of the SHS flux, $F$, on the concentration, at $50{ }^{\circ} \mathrm{C}$, in PAAm with different degrees of cross-linking: $(\square)$ $0.3 \%$; (O) $0.7 \%$; $\triangle$ ) $7.0 \%$.

between unimers and unimers/aggregates inside the matrix is not strong enough, leaving unimers free to permeate the membranes. We also point out that the fitting equation for the sorption isotherms are clearly dominated by the values of $C$ at higher $c$ values, especially in the case of SHS. In these circumstances the flux $F$ depends on the concentration of mobile dissolved species inside matrix.

The flux of different surfactants through polyacrylamide membranes shows a similar variation with the concentration. The cmc plays an important role in changing the permeability features of surfactants through polyacrylamide gels. In fact, although the micelles cannot enter or pass through the polyacrylamide membranes, as seen by fluorescence measurements reported in [6], a decrease is found in the slope of variation of $F$ with $c$ at concentrations above the $\mathrm{cmc}$. We may also note that at concentrations in the region of the $\mathrm{cmc}$ such relationships are non-linear.

In the case of the SOS, the permeability experiments were carried out at concentrations below the $\mathrm{cmc}$ and, for this reason, a unique linear relationship between $F$ and $c$ is found. Table 3 shows the fitting parameters of a linear equation that fits the experimental data of $F$ as a function of $c$, at concentrations range before and after the corresponding $\mathrm{cmc}$, together with the corresponding correlation coefficients.

\subsection{Diffusion coefficients of anionic surfactants in hydro- gels}

As discussed above, for the different surfactants, $C$ increase with $c$ in a multicomponent model way. The experimental results of sorption, in addition to the fluxes, show that so-called binding population is not completely immobilised and that no significant interference in the diffusion process occurs. This has been observed before $[13,14]$. Therefore, assuming that, (i) during steady-state surfactant permeation there is no significant swelling in 
Table 3

Linear regression for fitting the experimental data (Figs. 4-6) to $F=m c+y_{0}$

\begin{tabular}{|c|c|c|c|c|}
\hline Gel & $\Delta c(\mathrm{M})$ & $m(\mathrm{SE})\left(\mathrm{M}^{-1}\right)$ & $y_{0}(\mathrm{SE})\left(\mathrm{M} \mathrm{cm}^{2} \mathrm{~s}^{-1}\right)$ & $R^{2}$ \\
\hline \multicolumn{5}{|l|}{ SOS } \\
\hline I & $0-0.1$ & $2.13(0.01) \times 10^{-6}$ & 0 & 1.00 \\
\hline II & $0-0.1$ & $1.81(0.03) \times 10^{-6}$ & 0 & 1.00 \\
\hline III & $0-0.1$ & $6.29(0.01) \times 10^{-7}$ & 0 & 1.00 \\
\hline \multicolumn{5}{|c|}{$S D S$} \\
\hline \multirow[t]{2}{*}{ I } & $0-2 \times 10^{-3}$ & $3.54(0.03) \times 10^{-6}$ & 0 & 1.00 \\
\hline & $1 \times 10^{-2}-4 \times 10^{-2}$ & $7.09(0.37) \times 10^{-7}$ & $1.69(0.09) \times 10^{-8}$ & 1.00 \\
\hline \multirow[t]{2}{*}{ II } & $0-2 \times 10^{-3}$ & $1.16(0.06) \times 10^{-6}$ & 0 & 1.00 \\
\hline & $1 \times 10^{-2}-4 \times 10^{-2}$ & $2.98(0.16) \times 10^{-7}$ & $6.72(0.42) \times 10^{-9}$ & 1.00 \\
\hline \multirow[t]{2}{*}{ III } & $0-2 \times 10^{-3}$ & $6.29(0.05) \times 10^{-7}$ & 0 & 1.00 \\
\hline & $1 \times 10^{-2}-4 \times 10^{-2}$ & $7.12(0.63) \times 10^{-8}$ & $7.12(0.17) \times 10^{-9}$ & 1.00 \\
\hline \multicolumn{5}{|c|}{ SHS } \\
\hline \multirow[t]{2}{*}{ I } & $0-2 \times 10^{-4}$ & $1.08(0.02) \times 10^{-5}$ & 0 & 1.00 \\
\hline & $6 \times 10^{-4}-1 \times 10^{-3}$ & $3.75(0.23) \times 10^{-6}$ & $2.99(0.19) \times 10^{-9}$ & 1.00 \\
\hline \multirow[t]{2}{*}{ II } & $0-2 \times 10^{-4}$ & $1.03(0.02) \times 10^{-5}$ & 0 & 1.00 \\
\hline & $6 \times 10^{-4}-1 \times 10^{-3}$ & $3.52(0.07) \times 10^{-6}$ & $2.87(0.06) \times 10^{-9}$ & 1.00 \\
\hline \multirow[t]{2}{*}{ III } & $0-2 \times 10^{-4}$ & $8.62(0.56) \times 10^{-6}$ & 0 & 1.00 \\
\hline & $6 \times 10^{-4}-1 \times 10^{-3}$ & $3.15(0.06) \times 10^{-6}$ & $2.15(0.05) \times 10^{-9}$ & 1.00 \\
\hline
\end{tabular}

$\mathrm{SE}=$ standard error $(95 \%$ confidence interval $) ; R^{2}=$ correlation coefficient.

polyacrylamides, and (ii) the kinetics of formation and destruction of aggregates is faster than surfactant diffusion [15], Fick's law will mainly govern the process of diffusion. Consequently the integral diffusion coefficients, $D_{\mathrm{i}}$, of the different surfactants inside PAAm, can be computed by:

$D_{\mathrm{i}}=F / C$

Figs. 7-9 show the variation of the integral diffusion coefficients of the different surfactants, SOS, SDS and SHS, respectively, in the polyacrylamide gels with different degrees of cross-linking.

\subsubsection{Diffusion coefficients of SOS}

The variation of the diffusion coefficients of SOS with concentration shows a continuous increase of $D$ with

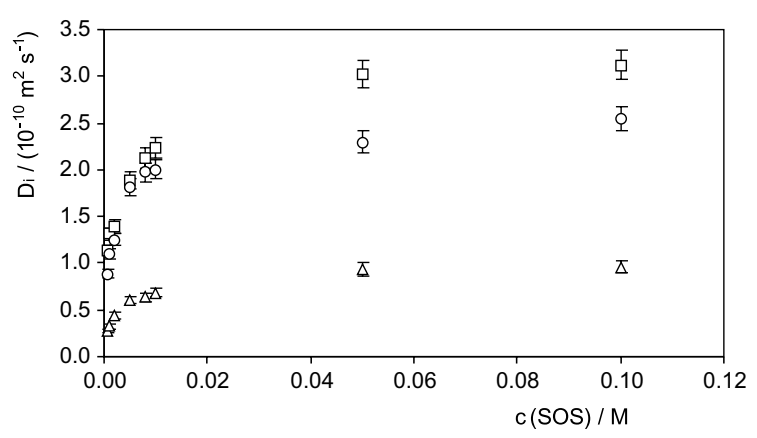

Fig. 7. Integral diffusion coefficients, $D_{\mathrm{i}}$, of SOS in PAAm with different degrees of cross-linking: $(\square) 0.3 \%$; (O) $0.7 \%$; $\triangle$ ) $7.0 \%$.

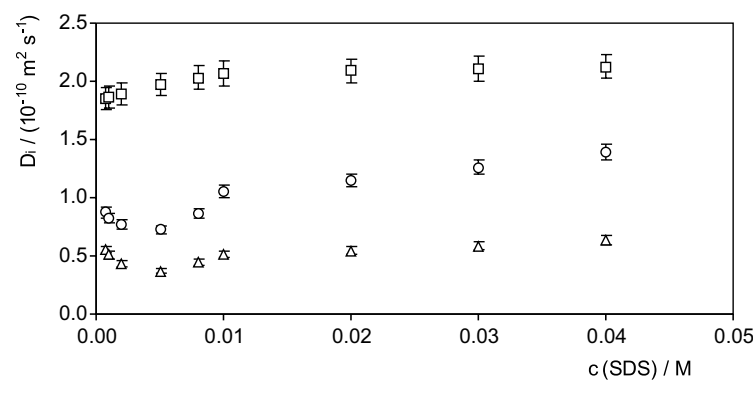

Fig. 8. Integral diffusion coefficients, $D_{\mathrm{i}}$, of SDS in PAAm with different degrees of cross-linking: $(\square) 0.3 \%$; (O) $0.7 \%$; $\triangle$ ( $7.0 \%$.

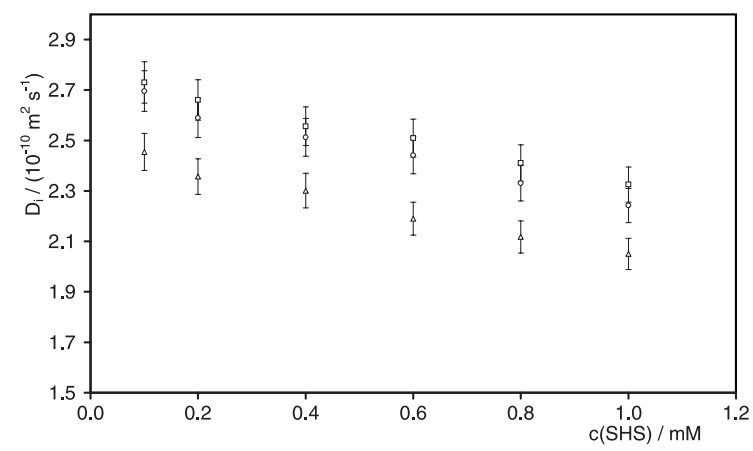

Fig. 9. Integral diffusion coefficients, $D_{\mathrm{i}}$, of SHS (at $50{ }^{\circ} \mathrm{C}$ ) in PAAm with different degrees of cross-linking: ( $\square$ ) $0.3 \%$; (O) $0.7 \% ;(\triangle) 7.0 \%$.

two clearly different slopes. Although the diffusion coefficients of SOS have been measured in a concentration 
range below the cmc, the higher values of $D_{i}$, to SOS concentrations higher than $0.05 \mathrm{M}$, are very similar to those in aqueous solutions. This concentration, three times lower than the cmc $(0.151 \mathrm{M}$ [16]) suggests that diffusing unimers are dissolving in that part of the water with a liquid-like structure.

\subsubsection{Diffusion coefficients of SDS}

Analysis of diffusion coefficients shows that the transport mechanism of SDS (Fig. 8) inside the gels is similar to that occurring with SOS: in the most hydrophilic gel (PA-I) the $D_{\mathrm{i}}$ values are of the same order of magnitude as surfactant mutual diffusion coefficients in aqueous solutions [17] and a clear plateau is observed at $C>$ $9.23 \times 10^{-3} \mathrm{M}$. Such a plateau can be due to the presence of aggregates $\left(\mathrm{cmc}=8.27 \times 10^{-3} \mathrm{M}\right.$ [3]. As the degree of cross-linking increases the concentration of SDS inside matrix where an inflexion point (of $D$ as a function of $c$ ) occurs also decrease to $4.02 \times 10^{-3}$ and $8.27 \times 10^{-4} \mathrm{M}$ to PA-II and PA-III, respectively), showing the increasingly important role of polymer chain packing and combined effect of the surfactant chain length and water content. This view is enhanced by the analysis of the variation of $D_{\mathrm{i}}$ of SDS in the less hydrophilic gels (PA-II and PA-III).

The mutual diffusion coefficients can be related to a frictional factor (which depends on the size of the diffusing molecules and viscosity) and an equilibrium thermodynamic factor for the change in chemical potential with concentration [17]. Therefore the decrease in $D_{\mathrm{i}}$ with surfactant concentration can be explained by a reduction in the thermodynamic factor (related to the variation of the activity coefficient with $c$ ), whilst the increase of $D$ with $c$ can be explained by a decrease of the resistance coefficient which is a measure of the friction acting on a solute as it moves through a solvent [18]. Such a decrease in the resistance coefficient could be explained by the formation of aggregates/micelles or/and the decreasing of the effective water free volume. Although the friction acting on a micellar cluster is much greater than that acting on a single surfactant unimer, on a per mole basis the transport in the micellar form is actually more efficient [19]. Thus, in general, the effect of water on SDS diffusion in the gel is different from that in aqueous solution: the presence of aggregates inside gels changes the diffusion coefficients very smoothly, whereas in aqueous solutions the presence of micelles leading to an approximately $70 \%$-decrease of diffusion coefficient values.

\subsubsection{Diffusion coefficients of SHS}

Following the variation of the integral diffusion coefficients of SHS with concentration (Fig. 9), SHS diffusion shows a very small decrease when concentration increases. Although the concentration range considered is different, the diffusion coefficients are slightly higher than the SDS values, which can be explained by the higher temperature at which the experiments were carried out. From comparison between SHS and other surfactants it is also possible to show that there is no significant effect of hydrogel swelling degree in the diffusion experiments. This can be justified in terms of the larger, and hence more hydrophobic, chain in the SHS.

\section{Theoretical approach to diffusion coefficients}

Different literature models for diffusion processes in homogeneous and heterogeneous gels [2] were tested. Figs. 10-12 show the variation of logarithm of diffusion coefficients as a function of polymer volume fraction. The regression results are given in Tables 4-6. $\varphi$ values were calculated using [20]

$\varphi=\{1+[(Q-1) \rho / d]\}^{-1}$

assuming that all sorbed surfactant has a density, $d$, similar to that in aqueous solutions [21]. The polymer densities, $\rho$, and the swelling degrees, $Q$, are taken from Table 1.

From the analysis of Tables 4-6, we may conclude that two of the reported equations are successful in interpreting the effect of cross-linker in the surfactant diffusion process: the free volume equation (original (Eq. (8)) [22] and modified by Peppas and Reinhart (Eq. (9)) [23]), and Cukier's equation based on a hydrodynamic model (Eq. (10)) [24].

As a first approximation, the free volume can be qualitatively visualized as the volume that is not occupied by the polymer molecules, but which constitutes a part of the bulk volume of the overall polymer/solvent or solution system. The free volume may be closely related to the void volume in semicrystalline polymers, and may be more generally visualized as a "hole", either

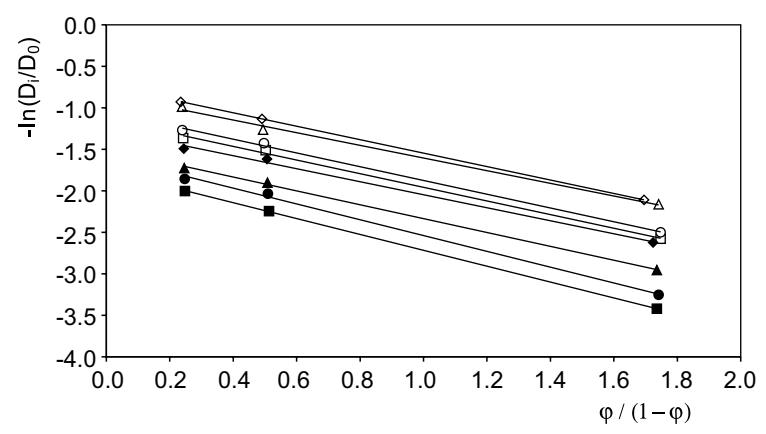

Fig. 10. Diffusion coefficients of SOS, $D_{\mathrm{i}}$, at different concentrations, as a function of polymer volume fraction, $\varphi$, in PAAm

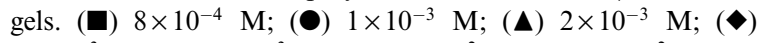
$5 \times 10^{-3} \mathrm{M} ;(\square) 8 \times 10^{-3} \mathrm{M} ;(\bigcirc) 1 \times 10^{-2} \mathrm{M} ;(\triangle) 5 \times 10^{-2} \mathrm{M} ;(\diamond)$ $1 \times 10^{-1} \mathrm{M}$. 

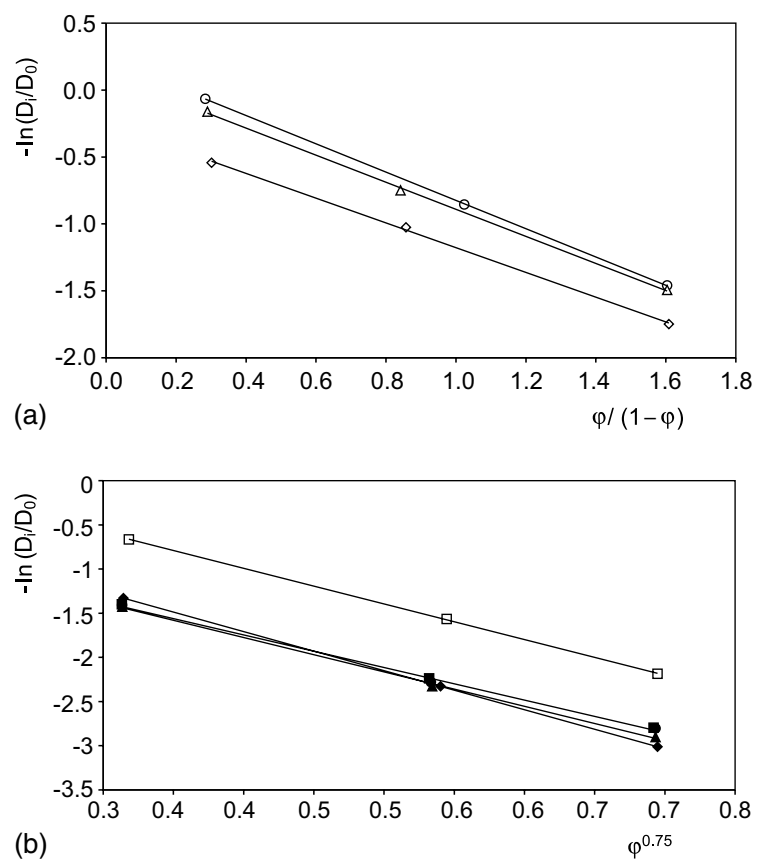

Fig. 11. (a) Diffusion coefficients of SDS, $D_{\mathrm{i}}$, at different concentrations, as a function of the polymer volume fraction, $\varphi$, in PAAm gels. (ם) $8 \times 10^{-4} \mathrm{M} ;(\bullet) 1 \times 10^{-3} \mathrm{M} ;(\boldsymbol{\Delta}) 2 \times 10^{-3} \mathrm{M} ;(\bullet)$ $5 \times 10^{-3} \mathrm{M}$; ( $\square$ ) $8 \times 10^{-3} \mathrm{M}$. (b) Diffusion coefficients of SDS, $D_{\mathrm{i}}$, at different concentrations, as a function of polymer volume fraction, $\varphi$, in PAAm gels. (O) $1 \times 10^{-2} \mathrm{M} ;(\triangle) 2 \times 10^{-2} \mathrm{M} ;(\diamond)$ $3 \times 10^{-1} \mathrm{M}$.

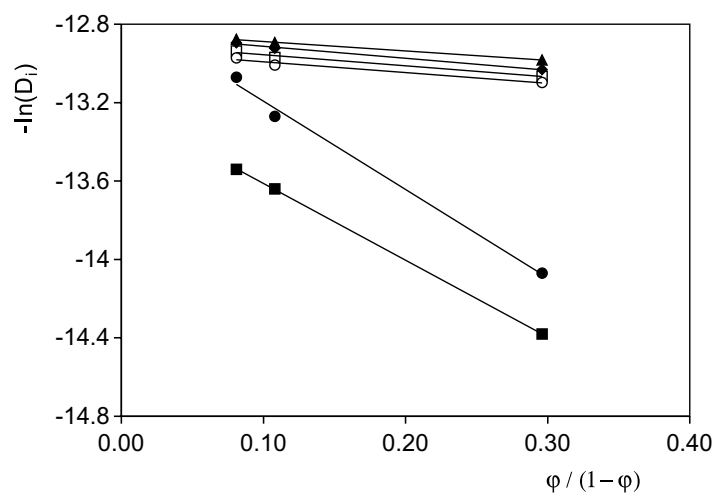

Fig. 12. Diffusion coefficients of SHS, $D_{\mathrm{i}}$, at different concentrations, as a function of polymer volume fraction, $\varphi$, in PAAm

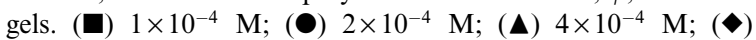
$6 \times 10^{-4} \mathrm{M}$; $(\square) 8 \times 10^{-4} \mathrm{M}$; (O) $1 \times 10^{-3} \mathrm{M}$.

opened up by thermal fluctuation of molecules or present because of geometrical requirements of random chain packing [22]. Using this concept the diffusion coefficient of a solute in a gel, $D$, can be expressed as follows
$D=D_{0} \exp (-k \varphi /(1-\varphi))$

where $D_{0}$ is the diffusion coefficient of the solute in aqueous solution, and $k$ is a constant related to the cross-sectional area of the diffusion solute and the free volume of water in the polymer. Peppas and Reinhart [23] suggested the equation

$\ln D / D_{0}=\ln k_{1}-k_{2}[\varphi /(1-\varphi)]$

where $k_{1}$ and $k_{2}\left(=k^{\prime} r_{\mathrm{s}}^{2}\right)$ are undefined structural constants for a given polymer-solvent system and $r_{\mathrm{s}}$ is the radius of the solute. The constant $k_{1}$ is, however, related to a sieving factor for diffusion in the polymer, when the volume of the solute becomes the critical geometrical parameter deciding whether it will pass through the polymer chains.

The hydrodynamic equation [24]

$D=D_{0} \exp \left(-k_{\mathrm{c}} r_{\mathrm{s}} \varphi^{0.75}\right)$

describes the diffusion in gels based in the concept that solute molecules are hard spheres which are large compared to the solvent molecules in which they move, and that the Brownian motion of these species can serve as probe of the nature of hydrodynamic screening in the solution; in Eq. (9), $k_{\mathrm{c}}$ is an undefined constant for a given polymer-solvent system.

The regression parameters of Eq. (8) or (9) for the experimental results shown in Figs. 10-12 are shown in Tables 4-6, respectively for SOS, SDS and SHS systems.

The mutual diffusion coefficients, $D_{0}$, used for Figs. 10-12, are taken from the literature [25]; since, as far as authors know, there is no available data for SHS we have plotted the experimental data in terms of $\ln \left(D_{\mathrm{i}}\right)$ as a function of $[\varphi /(1-\varphi)]$.

In terms of the free volume model, from Table 4 it can be seen that, with the possible exception of the two most dilute solutions, within the error limits there is no variation in the solute radius parameter, $k_{2}$. However, there is an increase, with concentration, of the screening effect of the polymer factor $\left(k_{1}\right)$, which approaches the value of 1 as the mesh size of the hydrogel, $\xi$, becomes much larger than the solute size, $r_{\mathrm{s}}\left(k_{1}=1-\left(r_{\mathrm{s}} / \xi\right)\right)$. How do we explain, therefore, such results if we expect an increase of the solute radius with the formation of aggregates? One possible explanation comes from consideration of the possible unimer-unimer, water-water and unimer-water interactions occurring in the matrix. In the presence of a unimer, the hydrophobic alkyl chain will provoke a rearrangement of water structure to produce a structure with lower free energy. This can be achieved, for example, by increasing the interactions between freezing and non-freezing water molecules. With an increase of unimers, some of these may interact to form aggregates. If these have a normal micellar structure, the ionic head groups will be in contact with water and be expected to lead to a decrease in the non-freezing water content with 
Table 4

Regression results of application of the free volume equation (9) of Peppas and Reinhart to surfactant diffusion in polyacrylamide gels

\begin{tabular}{lllll}
\hline$[\mathrm{SOS}] / \mathrm{M}$ & $\ln \left(k_{1}\right)(\mathrm{SE})$ & $k_{2}(\mathrm{SE})$ & $R^{2}$ & $k_{1}$ \\
\hline 0.0008 & $-1.762(0.008)$ & $0.956(0.007)$ & 1.000 & 0.172 \\
0.001 & $-1.588(0.047)$ & $0.952(0.045)$ & 0.999 & 0.204 \\
0.002 & $-1.501(0.029)$ & $0.835(0.027)$ & 0.999 & 0.223 \\
0.005 & $-1.264(0.053)$ & $0.783(0.051)$ & 0.998 & 0.283 \\
0.008 & $-1.135(0.046)$ & $0.822(0.043)$ & 0.999 & 0.321 \\
0.01 & $-1.045(0.038)$ & $0.830(0.036)$ & 0.999 & 0.431 \\
0.05 & $-0.841(0.055)$ & $0.763(0.052)$ & 0.998 & 0.466 \\
0.1 & $-0.763(0.001)$ & $0.810(0.001)$ & 1.000 & \\
\hline
\end{tabular}

$\mathrm{SE}=$ standard error $(95 \%$ confidence interval $) ; R^{2}=$ correlation coefficient.

Table 5

Regression results of application of the hydrodynamic (Eq. (10)) and free volume (Eq. (9)) equations to SDS diffusion in polyacrylamide gels

\begin{tabular}{|c|c|c|c|c|}
\hline$[\mathrm{SDS}] / \mathrm{M}$ & $\left(k_{\mathrm{c}} r_{\mathrm{s}}\right)(\mathrm{SE})$ & $y_{0}$ & $R^{2}$ & \\
\hline 0.0008 & $4.143(0.099)$ & & 1.000 & \\
\hline 0.001 & $4.172(0.103)$ & & 0.999 & \\
\hline 0.002 & $4.283(0.086)$ & & 0.999 & \\
\hline 0.005 & $4.313(0.022)$ & & 1.000 & \\
\hline 0.008 & $2.933(0.231)$ & & 0.919 & \\
\hline \multirow[t]{2}{*}{$0.008^{*}$} & $4.028(0.026)$ & $0.619(0.026)$ & 1.000 & \\
\hline & $\ln \left(k_{1}\right)(\mathrm{SE})$ & $k_{2}(\mathrm{SE})$ & & $k_{1}$ \\
\hline 0.01 & $0.233(0.038)$ & $1.058(0.006)$ & 1.000 & 1.262 \\
\hline 0.02 & $0.120(0.026)$ & $1.011(0.024)$ & 1.000 & 1.127 \\
\hline 0.04 & $-0.252(0.027)$ & $0.924(0.025)$ & 1.000 & 0.777 \\
\hline
\end{tabular}

${ }^{*}$ Regression parameters for an equation $\ln \left(D / D_{0}\right)=y_{0}-k_{\mathrm{c}} r_{\mathrm{s}} \varphi^{0.75} ; \mathrm{SE}=$ standard error $(95 \%$ confidence interval $) ; R^{2}=$ correlation coefficient.

Table 6

Regression results of application of the free volume equation (9) of Peppas and Reinhart to SHS diffusion in polyacrylamide gels, at $50{ }^{\circ} \mathrm{C}$

\begin{tabular}{llll}
\hline$[\mathrm{SHS}] / \mathrm{M}$ & $\ln \left(D_{0} k_{1}\right)(\mathrm{SE})$ & $k_{2}(\mathrm{SE})$ & $R^{2}$ \\
\hline 0.0001 & $-13.22(0.01)$ & $3.918(0.02)$ & 1.000 \\
0.0002 & $-12.74(0.01)$ & $4.50(0.34)$ & 0.997 \\
0.0004 & $-12.84(0.00)$ & $0.481(0.02)$ & 0.999 \\
0.0006 & $-12.85(0.01)$ & $0.611(0.05)$ & 0.997 \\
0.0008 & $-12.90(0.01)$ & $0.567(0.08)$ & 0.991 \\
0.001 & $-12.94(0.02)$ & $0.542(0.09)$ & 0.986 \\
\hline
\end{tabular}

$\mathrm{SE}=$ standard error $(95 \%$ confidence interval $) ; R^{2}=$ correlation coefficient.

a consequent increasing of the available "mesh size" for the diffusing process. In the SDS system at concentrations below the cmc, however, the diffusion fitted the hydrodynamic model better, suggesting that the decrease in the diffusion coefficient is essentially due to the frictional drag produced by polymer chains on the solute, whereas at concentrations above the $\mathrm{cmc}$, the free volume concept becomes relevant. The opposite occurs with
SOS. Whilst to the most hydrophilic and hydrophobic surfactants, SOS and SHS respectively, a single equation is used to model the experimental diffusion coefficients at the whole range of water free volume; in the SDS systems there is a transition in the diffusion mechanism at concentrations near the cmc, once neither Eq. (8) nor (9) give a good fit to the experimental results, suggesting that the hydrodynamic effect can be accompanied by a hindrance effect produced by the gel; the later becomes predominant at higher SDS concentrations.

The analysis of data in Table 6 and Fig. 12 shows two clear and different effects on the transport by diffusion of SHS through PAAm. At concentrations below or equal to $2 \times 10^{-4} \mathrm{M}$ the diffusion process is highly dependent on the free volume of the polymer, whereas at concentrations above this, the dependence decreases about an order of magnitude. Since $k_{2}$ depends both on a factor related to polymer-solvent interaction and also on solute radius, we believe that such a decrease in $k_{2}$ value can only be interpreted in terms of alterations of polymerwater interactions. That is, the presence of SHS at concentrations above $2 \times 10^{-4} \mathrm{M}$ leads to a rearrangement of the aqueous structure near polymeric that alters 
such interactions. Two different phenomena can occur: (a) one possibility is that SHS, having a hydrophobic chain, will interact (and partition) preferentially in a very structured medium, such as the non-freezing water [26], which is near the polar groups of the gel; under these circumstances such water structures act as a "trap", favouring the sorption of hydrophobic-type solutes [9]; (b) this sorption can occur independently or together with the formation of small aggregates. Consequently, we may suggest that the alteration of diffusing environment, for SHS/PAAm systems, occurs at $C$ between $4 \times 10^{-4}$ and $6 \times 10^{-4} \mathrm{M}$, and does not change with the uptake of water content of these gels. Assuming the mutual diffusion coefficients of SHS of the same order of magnitude of those of hexadecyltrimethylammonium bromide at $25^{\circ} \mathrm{C}\left(9 \times 10^{-6}-5 \times 10^{-6} \mathrm{~cm}^{2} \mathrm{~s}^{-1}\right)$, we find a $k_{1}$ value of approximately $0.4-0.6$. Although these values are lower than those obtained for the SDS, the free volume for diffusion in this case is also higher due to the increase of temperature. In any case, at $c>0.0004 \mathrm{M}$ the solute radius parameter as well as the sieving effect $\left(k_{1}\right)$ remains the same. This also suggests that the diffusing process mainly occurs via unimer.

\section{Conclusions}

In this work, the sorption of anionic surfactants was discussed on the basis of a multicomponent equation. The concentration of surfactant adsorbed in particular sites decrease with an increase of the surfactant chain length as well as with increase of cross-linker concentration. The equilibrium constant that characterise these sorption curves change in the opposite way. This suggests that the sorption of the surfactants is influenced by the hydrophobicity/hydrophilicity of surfactant structure, which show reflex in the steric hindrance $\left(C^{\prime}\right.$ values) as well as in the different interactions which take place with the different structures of the liquid water inside gel ( $K^{\prime}$ values). We also may point out that no specific interactions between ionic surfactants and the polymer does not occur at any polar polymeric group; this is supported for the following reasons: (i) the release of the sorbed surfactant is complete; (ii) comparing the experimental results of SHS with those found with hexadecyltrimethylammonium bromide (CTAB)/PAAm [27], we obtained $K^{\prime}$ values, for CTAB/PAAm systems, around 750 and the $C^{\prime}$ values obtained for SHS and CTAB with the same polymers were $0.007 / 0.008$ and $0.006 / 0.007 \mathrm{M}$, respectively; and (iii) there is no evidence for binding between neutral gels and anionic surfactants [14].

Various models have been tested for the diffusion behaviour in these systems. Of these, the free volume model, as presented by Peppas and Reinhart, and the hydrodynamic model of Cukier produce the best agreement with experimental. However, differences in the validity of these two models were observed, both in terms of the surfactant and the concentration range involved. It has been shown that the diffusion process of the most hydrophilic surfactant is clearly dominated by hydrodynamic factors; whilst to the more hydrophobic surfactants chain a molecular approach was more successfully. Although this approach can be found, very often, for small solute transport in the present case can be justified by a presence of a sieving effect provoked by the surfactants or by the polymeric network [28] on the transport phenomena; this is also supported by the fact that to SHS although the hydrophobicity is clearly higher, than the other surfactants considered, the polymer volume fraction is also lower $\left(0.07\right.$ and 0.23 at $50{ }^{\circ} \mathrm{C}$ compared with 0.2 and 0.6 at $25^{\circ} \mathrm{C}$ ) which also may contribute for the application of such model. Since these two models address different aspects of the diffusion of aggregating systems, such as surfactants, future studies will be aimed at further characterising the actual diffusing species.

\section{Acknowledgement}

Financial support from SAPIENS (POCTI/QUI/ $39593 / 2001)$ is gratefully acknowledged.

\section{References}

[1] Muhr A, Blanshard JMV. Diffusion in gels. Polymer 1982;23:1012-26.

[2] Amsden B. Solute diffusion within hydrogels. Mechanisms and models. Macromolecules 1998;31:8382-95.

[3] Jönsson B, Lindman B, Holmberg K, Kronberg B. Surfactants and polymers in aqueous solution. Chichester: John Wiley \& Sons; 1998.

[4] Hansson P. Surfactant self assembly in polyelectrolyte gels: aggregation numbers and their relation to the gel collapse and the appearance of ordered structures in the NaPA/ C $_{12}$ TAB system. Langmuir 1998;14:4059-64.

[5] Johansson L, Skantze U, Lofroth J-E. Diffusion and interactions in gels and solutions. 2. Experimental results on the obstruction effects. Macromolecules 1991;24:601923.

[6] Valente AJM, Burrows HD, Miguel MG, Lobo VMM. Diffusion coefficients of sodium dodecyl sulfate in water swollen cross-linked polyacrylamide membranes. Eur Polym J 2002;38:2187-96.

[7] Valente AJM, Polishchuk AYa, Lobo VMM, Burrows H. Transport properties of concentrated aqueous sodium dodecyl sulfate solutions in polymer membranes derived from cellulose esters. Langmuir 2000;16:6475-9.

[8] Tanford C. Physical chemistry of macromolecules. New York: John Wiley; 1961. p. 573-84.

[9] Lobo VMM, Valente AJM, Polishchuk AYa, Geuskens G. Transport of non-associated electrolytes in acrylamide hydrogels. J Mol Liq 2001;94:179-92. 
[10] Zheng X, Cao W. Interaction of main chain cationic polyelectrolyte with sodium dodecyl sulfate. Eur Polym J 2001;37:2259-62.

[11] Baker JP, Stephens DR, Blanch HW, Prausnitz JM. Swelling equilibria for acrylamide-based polyampholite hydrogels. Macromolecules 1992;25:1955-8.

[12] Frank HS, Franks F. Structural approach to the solvent power of water for hydrocarbons; urea as a structure breaker. J Chem Phys 1968;48:4746-57.

[13] Paul DR, Koros WJ. Effect of partially immobilizing sorption on permeability and the diffusion time lag. J Polym Sci, Polym Phys Ed 1976;14:675-8.

[14] Narita T, Gong JP, Osada Y. Kinetic study of surfactant binding into polymer gel-experimental and theoretical analyses. J Phys Chem B 1998;102:4566-72.

[15] Weinheimer RM, Evans DF, Cussler EL. Diffusion in surfactant solutions. J Coll Interf Sci 1981;80:357-63.

[16] Klevens HB. Critical micelle concentrations as determined by refraction. J Phys Coll Chem 1948;52:130-48.

[17] Deng Z, Lu H, Leaist DG. Mutual diffusion coefficients and resistance coefficients for aqueous solutions of sodium alkanoate surfactants. J Chem Eng Data 1996;41: 214-7.

[18] Tyrrell HJV, Harris KR. Diffusion in liquids. London: Butterworths; 1984. p. 52.

[19] Leaist DG. Binary diffusion of micellar electrolytes. J Coll Interf Sci 1986;55:230-40.
[20] Naghash HJ, Okay O. Formation and structure of polyacrylamide gels. J Appl Polym Sci 1996;60:971-9.

[21] Majer V, Roux A-L, Roux-Desgranges G, Viallard A. Rôle de lálcool dans le microemulsions. II. Volumes et capacities calorifiques molaires apparents du système eau+dodécylsulfate de sodium+isopropanol à $298,15 \mathrm{~K}$. Can J Chem 1983;61:139-46.

[22] Yasuda H, Lamaze CE, Ikenberry LD. Permeability of solutes through hydrated polymer membranes. Part I. Diffusion of sodium chloride. Makromol Chim 1968;118: 19-35.

[23] Peppas NA, Reinhart CT. Solute diffusion in swollen membranes. Part I. A new theory. J Membr Sci 1983;15: 275.

[24] Cukier RI. Diffusion of Brownian spheres in semidilute polymer solutions. Macromolecules 1984;17:252-5.

[25] Ribeiro ACF, Lobo VMM, Azevedo EFG, Miguel MG, Burrows H. Diffusion coefficients of sodium dodecylsulfate in aqueous solutions of sucrose and in aqueous solutions. J Mol Liq 2001;94:193-201.

[26] Ahluwalia JC. Thermodynamics of hydrophobic hydration. J Indian Chem Soc 1979;56:115-25.

[27] Valente AJM, Polishchuk AYa, Burrows H, Miguel MG, Lobo, VMM. Unpublished results.

[28] Amsden B. Solute diffusion in hydrogels: an examination of the retardation effect. Polym Gels Networks 1998;6:1343. 